\title{
Miniaturization of optical spectrometers
}

Authors: Zongyin Yang ${ }^{1,2 \dagger}$, Tom Albrow-Owen ${ }^{1 \dagger}$, Weiwei Cai ${ }^{4,5^{*}}$, Tawfique Hasan ${ }^{1,3^{*}}$

\section{Affiliations:}

${ }^{1}$ Department of Engineering, University of Cambridge, Cambridge CB3 0FA, UK

${ }^{2}$ College of Information Science \& Electronic Engineering, State Key Laboratory of Modern Optical Instrumentation, Zhejiang University, Hangzhou, 310027, China

${ }^{3}$ Cambridge Graphene Centre, University of Cambridge, Cambridge CB3 0FA, UK

${ }^{4}$ Key Laboratory of Education Ministry for Power Machinery and Engineering, School of Mechanical Engineering, Shanghai Jiao Tong University, Shanghai 200240, China

${ }^{5}$ Erlangen Graduate School in Advanced Optical Technologies (SAOT), Friedrich-AlexanderUniversität Erlangen-Nürnberg (FAU), 91052 Erlangen, Germany

*Correspondence to: cweiwei@sjtu.edu.cn, th270@cam.ac.uk

$\uparrow$ These authors contributed equally to this work

\begin{abstract}
Spectroscopic analysis is one of the most widely used analytical tools across both scientific research and industry. Whilst laboratory bench-top spectrometer systems offer superlative resolution and spectral range, their miniaturization is crucial for applications where portability is paramount, or in-situ measurements must be made. Advancement in this field over the last three decades is now yielding microspectrometers with performance and footprint near those viable for lab-on-a-chip systems, smartphones and other consumer technologies. In this review, we briefly summarize the technologies that have emerged toward achieving these aims including miniaturized dispersive optics, narrowband filter systems, Fourier transform interferometers and reconstructive microspectrometers - and discuss the challenges associated with improving spectral resolution while device dimensions shrink ever further.
\end{abstract}




\section{Main Text:}

Optical spectrometers have served as one of the most important instruments to date for materials characterization and chemical analysis (1). Conventional bench-top spectrometers typically rely on a combination of bulky dispersive optics, long optical path lengths, detector arrays and movable parts. These requirements impede their miniaturization for applications where it is critical to minimize size, cost and power consumption. Recent years have seen the development of scaled-down spectrometer systems for a wide range of handheld, portable and integrated applications, including in soil and crop analysis, monitoring of food industry production lines and marine/underwater scientific research (2-6). It is clear to see that in these uses, it is often far preferable to attain indicative, instantaneous and on-the-spot results, instead of transporting samples to a laboratory for ultra-high resolution analysis $(5,7)$. Further miniaturization, down to the sub-millimeter scale, could provide opportunities in a wide range of applications, including lab-on-a-chip spectroscopy and other in-situ or even in-vitro characterization systems. Various possibilities can be envisaged for consumer technologies, such as in smartphone-based devices $(8$, 9), for applications including the detection of counterfeit pharmaceuticals and banknotes, monitoring of skin health, or even determining the sugar and fat content in food products. On the other hand, in industry, devices suitable for drone-based spectral imaging - that is, where spectral information is correlated with spatial data - could revolutionize large-scale crop monitoring.

In general, reducing the size of a spectrometer necessitates a compromise with respect to degradation of its resolution, dynamic range or signal-to-noise ratio. However, microspectrometers can be engineered to meet 'acceptable' levels of performance for specific applications $(7,10)$. In many cases, where identification of signature spectral peaks, rather than relative metrology is concerned, a 'satisfactory' resolution in the visible range may, for instance, be in the order of 10 $\mathrm{nm}$, or even larger (5). Moreover, by enhancing a particular aspect of performance, microspectrometers can be specialized for extreme measurements that are challenging to implement using a conventional system. For example, the relative strengths of on-chip singlephoton spectrometers $(11,12)$ and single-nanowire spectrometers (13) lie in their ultra-high sensitivity detection and ultra-compact footprint, respectively.

Since the early 1990s, miniaturized optical spectrometers have been demonstrated based on a wide variety of designs and working principles, with a range of operational spectral bands and resolutions. In this review, we summarize the most explored technological platforms, presenting their relative merits and drawbacks. We have broadly organized the field into four main categories, based on the most distinct strategies for identifying different spectral components. First are those that feature dispersive optics to split light toward spatially separated detectors (Fig. 1A). Second, those that employ narrowband filters to preferentially transmit particular spectral components to different detectors (Fig. 1B). Third are Fourier Transform microspectrometers, based around temporal or spatial interferometers (Fig. 1C). Early miniaturized spectrometers fell within these first three classifications and featured designs that largely resembled scaled-down bench-top spectrometers, with out-of-plane diffractive optics or micro-electromechanical systems (MEMS)-based interferometers. Further development saw such designs largely give way to planar systems based on waveguides and integrated optics (14-19). However, in the last decade, taking advantage of more readily available computer processing power and reductions in microprocessor size and cost, a fourth category has emerged as a new paradigm of microspectrometer devices. 'Reconstructive' or 'computational' spectrometer systems (Fig. 1D) typically feature a set of 
detectors encoded with unique spectral response characteristics which, when measured in parallel, can be combined using complex algorithms to approximate or "reconstruct" an incident light spectrum. Such systems can harness not only technological advances in hardware, but also the development of new computational approaches, in particular, those based on compressive sensing and machine learning.

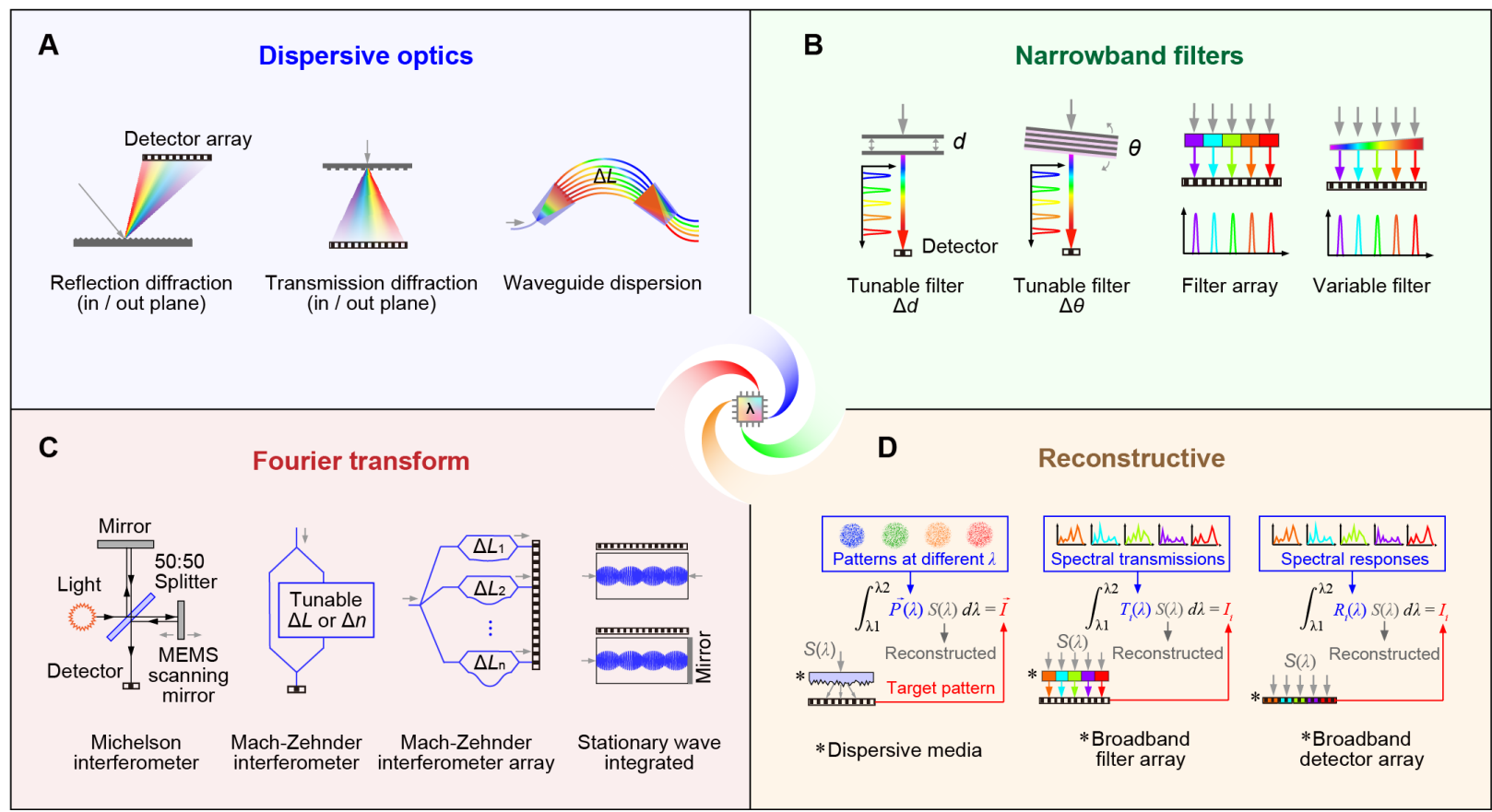

Fig. 1 Schematic summarizing the different underlying strategies for miniaturized spectrometer systems that have emerged over the last three decades. (A) Miniaturized dispersive optics. (B) Tunable or arrayed narrow-band filters. (C) Fourier transform and (D) Computational spectral reconstruction-based systems.

\section{Miniaturized dispersive optics}

Conventional spectrometers typically consist of one or several diffraction gratings, an optical path and a detector array. Light passes through an input slit and is collimated onto a diffraction grating which disperses spectral components in different directions. A concave mirror focuses this dispersed light toward the detector array. Advancements in micro- and nanofabrication techniques have provided an opportunity to develop microspectrometers by scaling down the components of these systems. A wide range of increasingly miniaturized, spatially 20 dispersive spectrometers with centimeter-scale footprints have been demonstrated since the 1990s $(20,21)$. These dispersive microspectrometers have typically been fabricated via wafer-bonding, with optical paths fashioned through electrochemically-controlled etching (Fig. 2A).

As the system footprint and thus component size decreases, a number of factors must be considered. Separation of spectral components at the detector plane depends on the distance light is allowed to travel after meeting the dispersive element. As such, for a given grating and detector array, the resolution, $\Delta \lambda$, (the full-width at half maximum (FWHM) of the narrowest 
distinguishable spectral component in nanometers) is proportional to the optical path length of the system. When the device is made more compact, the path length also necessarily decreases, thus lowering the spectral resolution. This can be compensated to an extent by increasing the detector's pixel density within a given width. However, the quality of optical components and the system alignment present an increasingly significant challenge from a fabrication standpoint; for instance, etching-induced surface roughness will cause more light to be scattered before arriving at the detector array (21).

Furthermore, the resolution will be impacted if there are no collimation components to image the input slit onto the detector. A feasible approach is to use a concave grating $(6,22,23)$; light is then dispersed and focused from different angles to different positions on the detector array without the need for complex collimation optics and multiple reflective components (Fig. 2B) (23). Commercial, manufacturable, visible-range microspectrometers based on this design have achieved a resolution of $\sim 10 \mathrm{~nm}$ with a footprint of $1-2$ centimeters (23). In addition to these concave gratings, a grating-Fresnel lens - an integrated combination of a diffraction grating and Fresnel lens - has been demonstrated as a diffractive optical element (24) within a smartphoneattached spectrometer system (Fig. 2C) (9).

Waveguides have been proposed as an alternative to free-space optics, allowing more compact light confinement, to further reduce footprint without significant compromise on performance (14). For this approach, input and output gratings were etched on the two sides of the substrate, followed by deposition of a waveguide layer on top. As illustrated in Fig. 2D, in these systems, light is coupled into the waveguide via an input grating. When propagating through the waveguide, light interacts with analytes on the waveguide surface. A photodetector array then detects the light on its exit from an output grating. As such, the system can be used to measure incident light spectra or the absorption spectra of the analytes on top of the waveguide through evanescent coupling.

These waveguide-based spectrometers have used various dispersion schemes (see Fig. 2EI), such as planar photonic crystals $(18,25)$, holographic elements (19), planar echelle gratings $(12,26)$, self-focusing phase transmission gratings (16), chirped grating (27) and arrayed waveguide gratings (AWG) (17), as well as metasurfaces (28). As with the out-of-plane devices, the resolution of waveguide-based spectrometers is inherently tied to the optical path length afforded by the system's footprint and as such, miniaturization necessitates a reduction in performance. Furthermore, with respect to manufacturability, fabrication tolerances (for example, in relation to sidewall-roughness induced losses) and waveguide mode coupling at low channel spacings present a challenge for extreme miniaturization below the millimeter scale $(29,30)$. However, aside from greater light confinement, advantages present themselves in their straightforward integration into monolithic, waveguide-based optical analysis systems.

In addition to innovations with respect to configuration and dispersive elements, simultaneously decreasing the size and increasing the sensitivity of the photodetector arrays provides another route towards smaller, higher performance microspectrometers. For example, superconducting nanowires have recently emerged as one of the most promising alternatives to semiconductor photodetectors for spectrometer applications, as they show ultrahigh sensitivities (single-photon detectability) with low jitter and dark counts $(11,12)$. Indeed, recent single-photon microspectrometer demonstrations using superconducting nanowires are capable of carrying out 
spectral analysis of ultra-faint light, which marks a step towards their use in astronomical spectroscopy and quantum computing $(11,12)$.

A

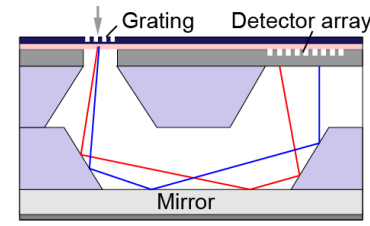

D

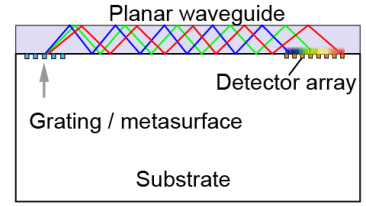

G

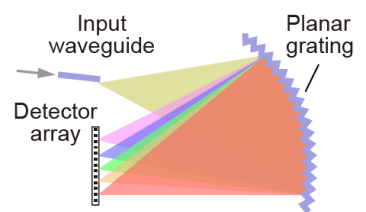

B

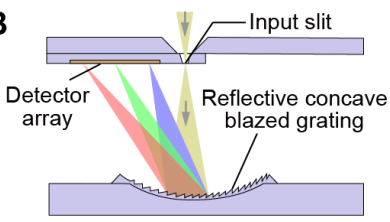

E

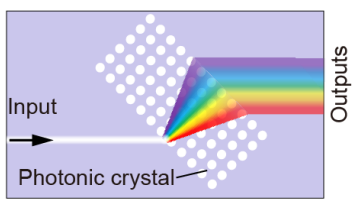

H

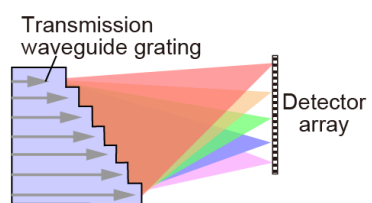

C

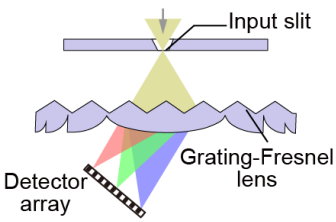

$\mathbf{F}$

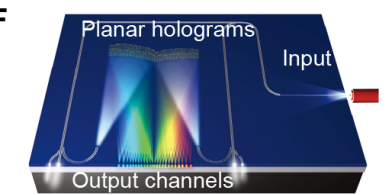

I

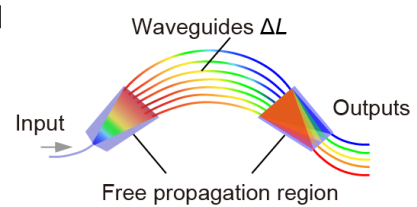

Figure 2. Spatially dispersive microspectrometers. (A) - (C) Miniaturized spectrometer systems based on out-of-plane spatial dispersion using planar (A) (21) and concave (B) (23) gratings. (C) Schematic of a grating-Fresnel spectrometer (9). (D) - (I) Waveguide based spectrometers based around different dispersion elements, showing (D) a buried grating on waveguide sensor (14), (E) a photonic crystal-based grating (18), (F) a holographic element (19), (G) a planar echelle grating (26), (H) a transmission waveguide grating (16) and (I) an arrayed waveguide grating (17). [(F) is adapted with permission from (19).]

\section{Narrowband filters}

Narrowband filters selectively transmit light with specific wavelengths, allowing for their use in spectrally-selective detection. In these systems, light dispersion can be achieved either with a single filter, the transmissive properties of which can be varied over time, or by passing light through an array of multiple unique narrowband filters each mounted onto their own detector. Whilst they are still limited by the detector and filter size, narrowband filter-based spectrometers offer a key advantage with respect to miniaturization over those based on dispersive systems. Aside from the benefit of their planarity, no separation (that is, path length) is required between the spectral filtering element(s) and the detector(s), circumventing one of the fundamental limitations of dispersive devices and affording the possibility of far more compact systems.

\section{Tunable filter-based microspectrometers}

A range of tunable narrowband filters, such as acousto-optic tunable (AOTFs) (31), liquidcrystal tunable (LCTFs) $(32)$, and Fabry-Pérot $(33,34)$ filters, as well as micro-ring resonators (35) have all been demonstrated in spectrometers. Their spectral transmission can be rapidly and dynamically controlled through the application of a voltage or acoustic signal, temporally separating spectral components. AOTFs use an acoustic field to generate a periodically fluctuating refractive index in solid-state birefringent crystals, analogous to a tunable diffraction grating. 
However, to date, size constraints on the birefringent crystals have presented a major obstacle to AOTF spectrometer miniaturization. LCTFs suffer from a similar constraint, containing a stack of polarizers and liquid crystal cells which are challenging to miniaturize. On the other hand, tunable Fabry-Pérot filters can be fabricated by well-established MEMS compatible processes (36), which make them highly suitable for microspectrometer mass production $(4,34)$.

A typical structure for a tunable Fabry-Pérot filter based microspectrometer (Fig. 3A) features a resonant optical cavity consisting of two parallel mirrors separated by a variable distance $d$. The transmission function $T$ of the Fabry-Pérot filter is given by the Airy-Function (37):

$$
T=\left(1-\frac{A}{1-r}\right)^{2} \frac{1}{1+\frac{4 r}{(1-r)^{2}} \sin ^{2}\left(\frac{2 \pi}{\lambda} n d \cos \theta-\varphi\right)}
$$

where $A$ is the absorbance of mirrors and cavity, $r$ is the reflectance of the mirrors, $n$ is the refractive index of the cavity medium, $\theta$ is the incidence angle and $\varphi$ is the phase shift at the reflectors, which normally is neglected. Light can be resonated and enhanced in the cavity when the optical distance between the two mirrors $(n d \cos \theta)$ is an integral multiple of its half wavelength $\lambda / 2$, which results in maximum ideal transmission through the cavity (and reflector) to the detector $(T=1)$. Figure $3 \mathrm{~A}$ shows $T$ as a function of light wavelength. Assuming that the absorbance and reflectance are fixed for a given device, during operation, transmission spectra can be tuned by varying the optical path length, which can be achieved by changing $n, d$ or $\theta$. Tuning the separation of the mirrors $(\Delta d)$ is the most common strategy, through the use of electrostatic or piezoelectric actuator (see Figs. 3B, C), where current MEMS technology straightforwardly affords a wide tunable range $(33,34,37)$. Tunability of the refractive index, $\Delta n$ can be realized by choosing electro-optically tunable $\mathrm{LiNbO}_{3}(38,39)$ or liquid crystals $(40)$ as the cavity medium, while varying the angle of incidence, $\Delta \theta$ has been demonstrated via rotating the filter (41).

There are certain key factors to consider in engineering these Fabry-Pérot spectrometers for high performance. The FWHM of transmission peaks determines the spectral resolution of the filter-based spectrometers, which in turn is equal to the intrinsic finesse of the cavity $F_{\text {int }}=\pi \sqrt{ } r$ / $(1-r)$ (20). Thus, a high resolution requires high reflectance, but for a metallic mirror cavity this results in lower transmission and therefore a weaker signal-to-noise ratio (34). A partial solution here is to use distributed Bragg reflectors (DBRs) as mirrors (42), which consist of alternating high and low refractive index dielectric quarterwave layers with high reflectance and low absorption at a specific spectral range. However, they are costly and significantly more complex to manufacture. Note that defects in the cavity system, including non-parallelism or mirror imperfections, also reduce the effective finesse (and thus resolution): $1 / F_{\text {eff }}^{2}=1 / F_{\text {int }}{ }^{2}+1 / F_{D}{ }^{2}$ where $F_{D}$ represents the defect finesse (20).

\section{Narrowband filter arrays and linear variable filters}

For the tunable narrowband filter spectrometers discussed above, the spectra are analyzed in a time sequence, sacrificing time response. This also presents an obstacle for high-speed spectroscopy applications. Narrowband filter arrays and linear variable filters offer an advantage in allowing simultaneous measurement of multiple spectral components in parallel (though this, in turn, necessitates multiple detectors). 
A
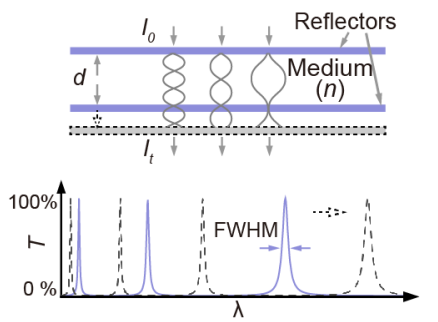

D

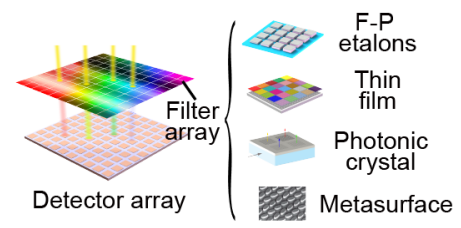

$\mathbf{F}$

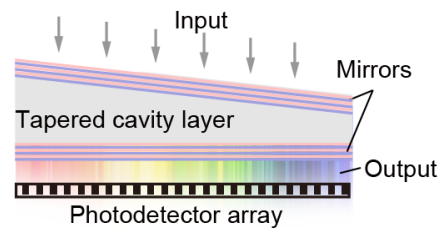

B

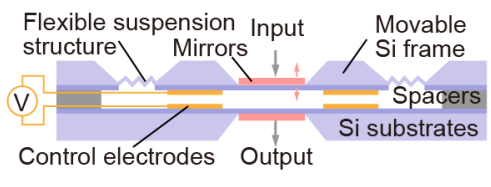

C

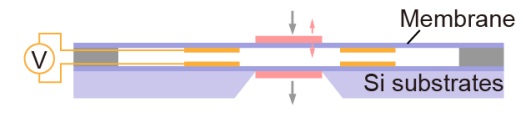

E

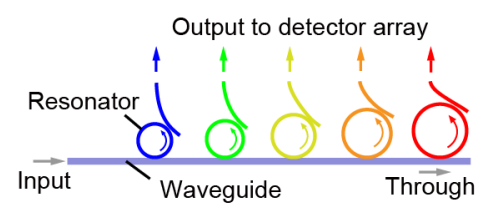

G

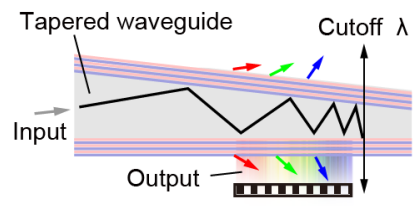

Figure 3. Narrow-band filter spectrometers. (A) Schematic configuration (top) and the corresponding transmittance function (bottom) of a typical Fabry-Pérot filter. (B), (C) Typical configurations of tunable Fabry-Pérot filter based microspectrometers showing (B) a bulk wafer bonding structure (43) and (C) a membrane-on-wafer structure (4). (D) Schematic of a filter array microspectrometer scheme based around Fabry-Pérot etalons (44), etched thin-films (45), photonic crystals (46) and metasurface (47). (E) A waveguide ring-resonator based filter strategy. (F), (G) Schematic of linear variable filter based spectrometer designs and their implementation, for $(\mathrm{F})$ transverse incidence $(48)$ and $(\mathrm{G})$ waveguided operation (49). [Bottom inset of 3(D) adapted with permission from (47).]

Fixed filter arrays have been exploited in many microspectrometers, where each filter is responsible for transmitting a specific wavelength onto the photodetector underneath. Various filter schemes exist, differentiated by their working principles, configurations and materials (50). Filter arrays based on Fabry-Pérot etalons (44), thin-films (45), planar photonic crystals (46), photonic bandgap fibers (51), metasurfaces (47) and waveguide ring resonators (35) have been demonstrated for the development of microspectrometers (see Figs. 3D, E). Clearly, the number of channels directly constrains the spectral resolution (in the case of the 16 filters in (44), for example, to $\sim 25 \mathrm{~nm}$ ) so processes which necessitate the individual placement or deposition of each filter are ill-suited. To address this, a combinatorial deposition technique can be used to fabricate a large number of filters in fewer steps; for example, an $8 \times 16$ array (128 channels) can be fabricated in only 9 deposition processes (45).

Another strategy to increase the ease of fabricating a wide range of transmission wavelengths is to use a linear variable filter, which is typically either a wedged (52) or composition-graded filter (53), where the transmission or reflection spectrum varies continuously along one axis of the filter. One approach here is to simply scan a spectrum by sliding the filter over a single detector (54). However, this is relatively slow and necessitates extra moving parts. Combining a linear variable filter with a detector array for parallel measurement offers an ideal 
solution (55). Microspectrometers based on this configuration have already been demonstrated, as shown in Fig. 3F (48). Tapered Bragg waveguides exhibit similar linear variable filtering capability due to the variable cutoff propagation wavelength, as depicted in Fig. 3G (49). Both linear variable configurations show high spectral resolution $(\sim 1 \mathrm{~nm})$.

\section{Fourier transform microspectrometers}

Typically used for absorption or emission spectroscopy in the infrared range, Fourier transform (FT) spectrometers center around the use of an interferometer to modulate the light incident on a single detector over time. The 'interferograms' collected at the detector (functions of received signal intensity over time, or a time-variant property of the system such as optical path length) are then converted to a wavelength-dependent spectrum via FT. FT systems have two main inherent benefits over those based on diffractive optics; firstly, collecting spectral information at one detector simultaneously results in the multiplex (or Fellgett's) advantage. Secondly, avoiding spatial dispersion results in a higher optical throughput, or étendue, known as Jacquinot's advantage. Both of these factors tend toward affording a higher signal-to-noise ratio. Moreover, using one detector offers a smaller and more cost-effective alternative to array-based detectors.

Miniaturized FT spectrometers can be categorized through the mechanism by which the optical path lengths within the interferometer are changed over time; the first broad differentiation being those with and without moving parts. Belonging to the former group, the earliest, as well as some of the more recent, chip-based FT spectrometers, appearing in the late 1990's, were based around Michelson interferometers, with MEMS employed to manipulate mirrors with either electrostatic $(56,57)$, electromagnetic (58) or electrothermal (59) (often comb-drive) actuators; see Fig. 4A. Aside from difficulties in integration with planar light sources, a drawback of these devices is that the spectral resolution is limited by the maximum optical path length difference (OPD) allowed by the actuator travel range (60), which in turn is constrained by, for instance, the pull-in effect (whereby, below a threshold separation, the mirrors will uncontrollably and rapidly attract together) $(2,6)$. Very recently, it has been demonstrated that the evaporation of a droplet atop the end of an optical fiber can also function as a system analogous to a scanning FT spectrometer, to obtain the absorption spectra of liquid analytes (61).

As of the late 2000s, planar on-chip FT spectrometers, based on integrated waveguides and without movable mirrors - have emerged. Instead of Michelson architectures, these systems are based around Mach-Zehnder interferometers (MZIs) whereby light is split into at least two unidirectional pathways toward a single detector where they are recombined. Here, the OPD induces a phase difference between light in different channels. Some of these systems are based around arrays of multiple MZIs, forming spatial heterodyne spectrometers (SHSs) (62-64). For instance, an array of spirally-coiled waveguides were fabricated (Fig. 4B), with their length varying linearly by $\Delta L$, inducing a delay between the paths (63). However, such a system is limited by the maximum OPD, as well as the number of MZIs, both of which constrain the extent to which the spectrometer footprint can be reduced whilst maintaining satisfactory performance.

Building on this, alternative designs employ mechanisms which can continuously tune the characteristics of a single MZI (and thus requiring only a single photodetector) rather than relying on multiple MZIs with fixed lengths. This has been achieved through electro-optical modulation in, for example, $\mathrm{LiNbO}_{3}$ waveguides (60) as well as through exploiting thermo-optical effects, 
where microheaters are embedded adjacent to integrated optical pathways $(65,66)$ (Fig. 4C). An alternative approach is a digital FT spectrometer, where a photonic circuit is used, featuring a number of optical switches which divert the signal along paths of different lengths (67). Here, the resolution depends on the number of paths, or spectral channel count, which scales exponentially with the number of optical switches. In many of these methods, recent advances have allowed computational techniques, such as compressive sensing (64), machine learning (68) and forwardbackward linear prediction (60) to enhance the spectral resolution of these devices and correct for temperature change- or fabrication-based errors.

A related variant on these miniaturized interferometer-based FT systems is stationary wave integrated Fourier transform spectrometry (SWIFTS). In recent works on SWIFTS-based microspectrometers, a standing wave is set up within a single-mode, closed-loop waveguide through the interference of two counter-propagating signals (alternatively, in a Lippmann configuration, a mirror can be placed at the end of a waveguide to set up a standing wave by reflecting the signal back upon itself) $(69,70)$. Whilst temporal interferograms are produced and collected at a single detector in the previously mentioned FT spectrometers, here, a spatial interferogram is produced. Metallic nanoribbons are deposited with a regular spacing on top of the waveguide to sample the evanescent field and map the relative intensity of the standing wave along the loop (Fig. 4D). A proof of concept demonstration for this design acknowledged that the spectral range in such a system is constrained (in this case to $96 \mathrm{~nm}$ centered at $1500 \mathrm{~nm}$ ) as the samplers cannot be fabricated at a pitch to avoid undersampling of the interferogram (69).

However, more recently, a SWIFTS system has been developed which circumvents this issue using a dual, spatial and temporal sampling scheme. Use of the electro-optic effect in a hybrid $\mathrm{LiNbO}_{3}-\mathrm{SiN}$ waveguide platform allows the spatial interferogram to be shifted along the waveguide by applying a voltage (Fig. 4D), such that, even with a fixed array of nanosamplers, the whole interferogram can be sampled, in this case achieving a spectral range of $500 \mathrm{~nm}(71)$. The resolving power, $R=\lambda / \Delta \lambda$, in these devices is given by $2 n L / \lambda$, where $n$ is the refractive index of the waveguide, $L$ the length of waveguide being probed, and $\lambda$ the wavelength. As such, extremely high resolutions (tens of picometers) are possible over only centimeter length scales, though, conversely, such devices may not be suitable for extreme miniaturization. These systems also currently rely on an external camera to image the interferogram scattering from the samplers; the development of specialized nanoscale-photodetectors, to be placed on top of the waveguide, would allow for direct measurement and a simplified system. 
A

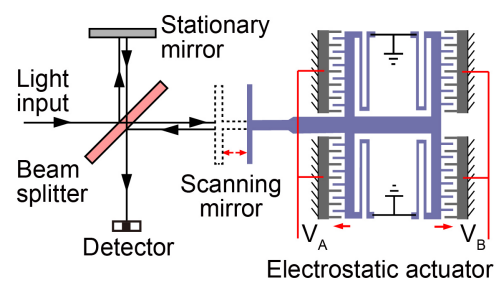

B

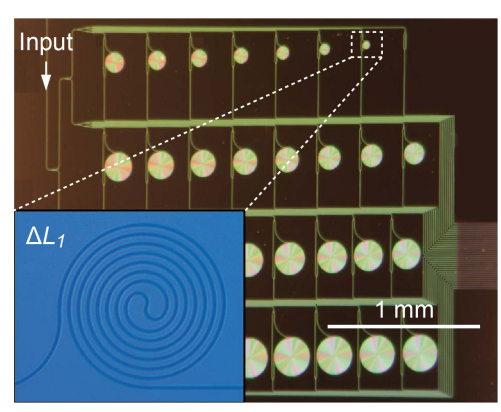

C

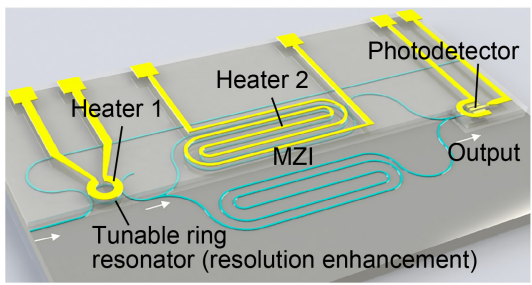

D

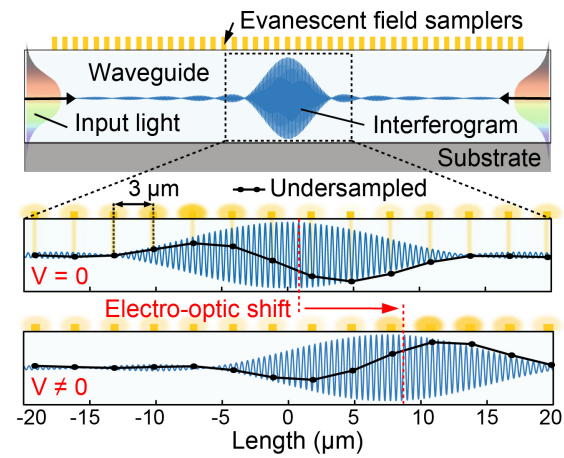

Figure 4. Fourier Transform microspectrometer platforms. (A) Schematic of a miniaturized, MEMS FT spectrometer based on a Michelson interferometer. The path lengths of the interferometer are varied over time via the electrostatic actuator to collect an interferogram which is subsequently converted to spectral data by Fourier transform (56). (B), (C) MZI-based, on-chip Fourier transform spectrometers. (B) Optical image of an SHS device based on an array of multiple MZIs with different OPDs, each varying by $\Delta L_{i}$. (C) Schematic of a microspectrometer based on a single MZI, using a micro-ring resonator (MRR) for resolution enhancement and a heater to facilitate path length modulation by thermo-optical effect. [(B) and (C) are adapted with permission from $(63,66)$.] (D) Schematic (top) of a standing-wave integrated Fourier transform spectrometer system in an electro-optically tunable medium; diagrams (below) illustrate the use of the electro-optic effect in $\mathrm{LiNbO}_{3}$ to shift the interferogram along the waveguide over time through application of a bias, $V$. [(D) is adapted with permission from (71).]

\section{Reconstructive spectrometers}

Over the past decade, a new spectrometer paradigm has emerged, which, as indicated by the name, relies on computational techniques to approximate or 'reconstruct' an incident light spectra from pre-calibrated information encoded within a set of detectors. More specifically, the 'reconstruction' here refers to the solution of a linear equation system. Thus far, two strategies have generally been seen for encoding spectral information within a set of detectors: complex spectral-to-spatial mapping and spectral response engineering.

\section{Complex spectral-to-spatial mapping}

In a conventional grating-based spectrometer, a point (i.e. a wavelength) in the spectral domain is mapped to a point (i.e. a detector) in the spatial domain (72). The readout of the detectors directly constitutes the spectrum. However, as mentioned previously, the spectral resolution scales with the distance from the grating to the detectors (i.e. the path length); one-to-one spectral-tospatial mapping is thus highly limited when looking to increase spectral resolution with a decreasing footprint (73). Complex spectral-to-spatial mapping is an alternative approach that 
distinguishes the wavelengths by creating a signature pattern (either 1D or 2D) in the spatial domain for each of the wavelengths (Fig. 5A). For example, when monochromatic light passes through a dispersive element such as a long multimode fiber (MMF), it will create a wavelengthdependent signature pattern at the output of the fiber due to the interference between the guided modes in the fiber (72). Thus, when passing an arbitrary polychromatic light through the MMF, the output will be the overlay of scaled signature patterns created by each individual wavelengths. The target spectrum to be reconstructed is essentially the assembly of scaling weights corresponding to these patterns (73). The signature patterns can also be generated by feeding the light into a miniaturized optical element such as a disordered photonic chip $(73,74)$, a spiral waveguide (75), a dispersive hole array (76), or a polychromator (77) (Figs. 5B-E). For a sought spectrum, $\vec{S}$, with normalized signature patterns, $\vec{p}\left(\lambda_{i}\right)$, and signals measured at the detector array, $\vec{I}$, the mapping process can be mathematically described as:

$$
\vec{I}=\sum_{k=1}^{K} \vec{p}\left(\lambda_{k}\right) \cdot S_{k}
$$

where $k$ enumerates a total number of $K$ signature patterns and the same number of elements in $S$.

Equation (2) can be discretized as:

$$
\vec{I}=P \cdot \vec{S}
$$

where $P$ is the mapping matrix, the columns of which correspond to the patterns.

If the signature patterns of two distinct wavelengths are identical, it is impossible to tell which wavelength leads to the measured pattern. In this way, the dissimilarity between the signature patterns determines the resolving power of the reconstructive spectrometers. Mathematically, the signature patterns are the columns of the matrix $P$; and the higher the similarity between the signature patterns, the larger the condition number of $P$, leading to poorer numerical solution of Equation (3). As a result, the signature patterns should be as diverse as possible. It has been recognized that the diversity is proportional to the spread of the optical path length i.e. the difference between the shortest and longest optical paths of the propagation modes within the chosen optical elements (73). A resolution of $0.01 \mathrm{~nm}$ has been demonstrated with a multi-mode spiral waveguide which utilizes evanescent coupling to significantly enhance the optical path length spread (75). Such a high resolution can greatly broaden the applications of miniaturized spectrometers. In addition, these systems are robust with respect to fabrication imperfections, which can be compensated for through calibration (75). However, they suffer from temperature variations which can change the signature pattern for a specific wavelength. The higher the spectral resolution, the more the spectrometer suffers from thermal instability. Thus, additional measures such as adding a temperature controller or temperature-dependent calibration should be adopted (75). Furthermore, the computational cost scales up with the spectral resolution for a fixed spectral range, as more variables need to be solved from an increased number of equations.

\section{Spectral Response Engineering}

The second approach to realize reconstructive spectrometers is to tailor a distinct spectral response for each of the detectors. This can be achieved either by engineering the detectors themselves or the optical elements integrated on top of the detectors (Fig. 5F). Assuming the spectral response of the $i$-th detector to be $\vec{T}_{i}(\lambda)$, its measured signal can be described as: 


$$
I_{i}=\int_{\lambda_{1}}^{\lambda_{2}} T_{i}(\lambda) \cdot S(\lambda) d \lambda
$$

The signals of the detector array can then be summarized in a discrete format as:

$$
\vec{I}=T \cdot \vec{S}
$$

where $T$ is a matrix, the rows of which corresponds to the detectors' spectral response functions.

By solving Equation (5), the unknown target spectrum can be reconstructed. The entire process is illustrated by Fig. 5F. Almost any optical element which can generate diverse spectral response functions can be adopted for spectrometry systems based on this principle such as quantum dots $(78,79)$ and photonic crystal slabs $(80)$ (Figs. 5G, H). Other designs including liquid crystals $(81)$, etalon arrays $(82)$, nanostructured photodiodes $(83,84)$, metasurfaces $(85)$ have also been used as the basis for such strategies. However, all these designs require a separate fabrication of both filter and detector arrays. This increases the complexity of manufacturing and limits miniaturization. Recently, spectral responsivity engineered nanostructure has been demonstrated to integrate both these functions $(13,86-90)$ as shown in Figs. 5I and J. For example, a computational spectrometer based on a composition-gradient alloyed semiconductor nanowire was proposed, which can be divided into a number of sections (detectors) along the axial direction (13). The response functions of these detectors vary due to the gradual and subtle variation in the alloy compositions. Whilst resolution is still modest $(\sim 5-10 \mathrm{~nm})$, such a nanowire spectrometer integrates the functions of both wavelength selectivity and photodetection into an individual nanostructure, pushing the footprint towards tens of microns, two orders of magnitude below that of any other computational spectrometer system.

However, as the size of the spectrometer decreases, the number of detectors (i.e. the number of equations) that can be accommodated also reduces due to the physical constraints, affecting the ultimate spectral resolution that can be achieved. In addition, the minimized footprint of the spectrometer reduces the light-matter interaction, compromising their sensitivity. In a follow-up work, a nanowire spectrometer was developed that can operate in a waveguide mode, providing an improved signal-to-noise ratio for such an ultra-compact device (87). 
A

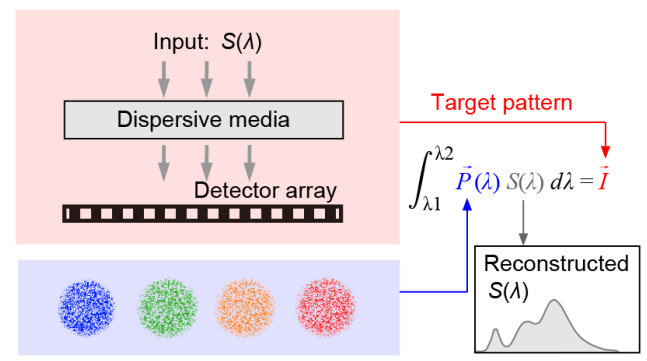

F

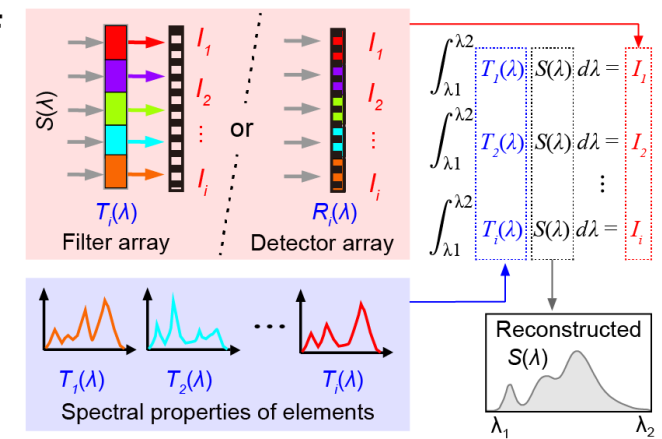

B

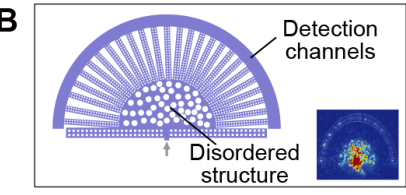

D

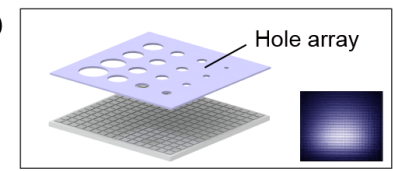

$\mathbf{G}$

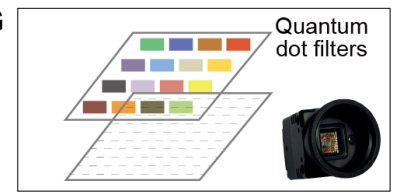

I

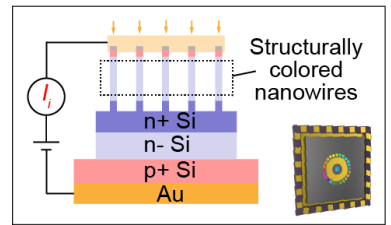

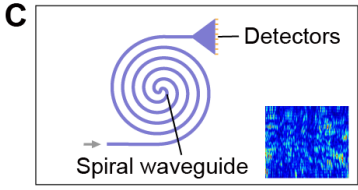
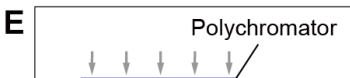

$\lim _{t}+4$ שחים
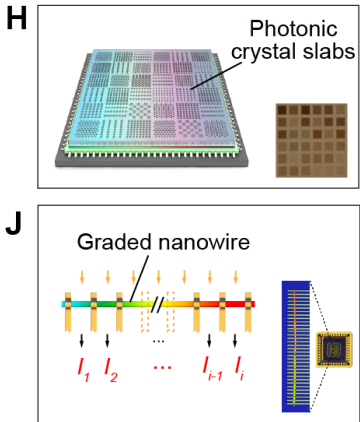

Figure 5. Computational microspectrometers. (A) Operational principles behind spectrometers based on spectral-to-spatial mapping. (B) - (E) Examples of optical systems that had been adopted for complex spectral-to-spatial mapping: (B) a disordered photonic chip, (C) a spiral waveguide, (D) a dispersive hole array, or (E) a polychromator. [(B) - (E) are adapted with permission from (73, 75-77)]. (F) Principles behind reconstructive spectrometers based on engineered spectral response filtering or detection. $(\mathrm{G})-(\mathrm{J})$ Examples of different reconstructive systems using spectrally-engineered filtering, with arrays based on (G) colloidal quantum dot mixtures and $(\mathrm{H})$ photonic crystal slabs, and spectrally-engineered detection, based on (I) arrays of structurally colored nanowires, and $(\mathrm{J})$ a single, compositionally engineered nanowire. $[(\mathrm{G})-(\mathrm{J})$ are adapted with permission from $(78)(80)(86)(13)]$.

\section{Reconstruction techniques}

The inversion problems presented in the two previous sub-sections are typically ill-posed. Different strategies must be adapted to alleviate this, depending on whether the problem is overdetermined or under-determined, that is, whether the number of detectors (and thus, spectral response functions) is higher or lower than the number of data points in the reconstructed spectrum, respectively. In the over-determined case, noise in the measurements will be readily amplified into reconstruction errors (91). To mitigate this, truncated singular value decomposition can be adopted to remove some part of the measured information which is most easily affected by the noise (92). When the problem is under-determined, additional information such as smoothness should be incorporated to find the most 'plausible' solution based on prior knowledge of the type of spectrum being measured (93). For example, the original spectrum can be approximated as the combination of a set of smooth basis functions such as Gaussian curves. Compressive sensing has also been adopted to explore the sparseness of the sought spectrum (94). According to compressive sensing theory, randomly structured response functions are preferred to improve the spectral resolution as the correlation between the functions is minimized (80). Dictionary/machine-learning techniques 
(95) are also promising alternatives to incorporate prior knowledge into the spectral reconstruction process.

\section{Summary and discussion}

We have summarized the technological evolution of miniaturized spectrometers, detailing their working principles and merits, under four broad classifications. Despite sharing similar overarching aims, works from these four sub-fields encompass a hugely diverse range of device designs and operational strategies. Drawing together the field as a whole, Fig. 6A compares the resolution, operational wavelength range and footprint across the four sub-categorizations of device strategies defined in each section. Clearly, there is a wide variance in performance, footprint and operational range - even within each sub-field - and no platform yet combines high resolution $(<1 \mathrm{~nm})$, wide spectral range $(>300 \mathrm{~nm})$ with ultra-compact $(<100 \mu \mathrm{m})$ physical dimensions. Behind these metrics, it is important to also consider the suitability and ease of integration of each devices for different application spaces. For instance, while the MEMS-based system in ref. (96) shows competitive resolution performance, wide spectral range and a small footprint, its complex, $400 \mu \mathrm{m}$-tall structure would prove highly challenging for integration into spectrometer arrays such as those required in the snapshot hyper-spectral imaging cameras discussed later in this section.

Additionally, the sensitivity of each device design is an increasingly important factor as the detector, and thus photon collection area, is reduced further in size. This is especially significant given many portable applications will rely on the collection of ambient light. Here, there are many common factors between the four categories above. For instance, the non-integrated systems discussed above can benefit from the introduction of a lens to focus light onto the detectors, filter array or dispersive medium $(78,97)$, while enhancing the SNR of the detectors is also one clear avenue for improvement (5). However, there are also a variety of distinct considerations dependent on the device design. In AWG systems for instance, the overall sensitivity is heavily influenced by the efficiency of coupling light into the waveguide, as well as the dispersion and propagation losses (15). As discussed previously, while FT systems benefit from a multiplex advantage in the use of only one detector, transmittance from for instance, a Fabry-Pérot cavity can be as low as $15 \%$ of the incident light (98).

Given the need for these devices to function outside controlled laboratory settings, the robustness and stability with respect to their external environment is another operational consideration. In this respect, there are two main impinging factors: changes in temperature and air composition (for instance, the impact of moisture). Thermal effects are of particular importance where the system is highly sensitive to changes in the refractive index of the active media, for example, in integrated systems such as AWG's, MZI's and SWIFTS's. Likewise, challenges can arise from the thermal expansion of gratings or MEMS components, as well as through temperature-dependencies in the spectral response function of the detectors in computational spectrometers. In many cases, temperature sensitive calibration can be applied to effectively eliminate (99) such effects, while hermetic sealing (3) or passivating coatings (13) can also be $40 \quad$ applied to stabilize device performance.

Finally, the relative maturity of these sub-fields must be taken into account when considering their prospects. To this end, Fig. 6B displays a timeline illustrating the emergence of 
key design innovations. As may be expected, these milestones have followed wider technological trends; early microspectrometers mainly took advantage of breakthroughs in micro-fabrication between the 1980's through to the early 2000's; advances in lithographic and etch processes, as well as the continued development of MEMS technology and waveguide-based chips, afforded the production of complex miniaturized dispersive, Fourier transform or filter-based systems.

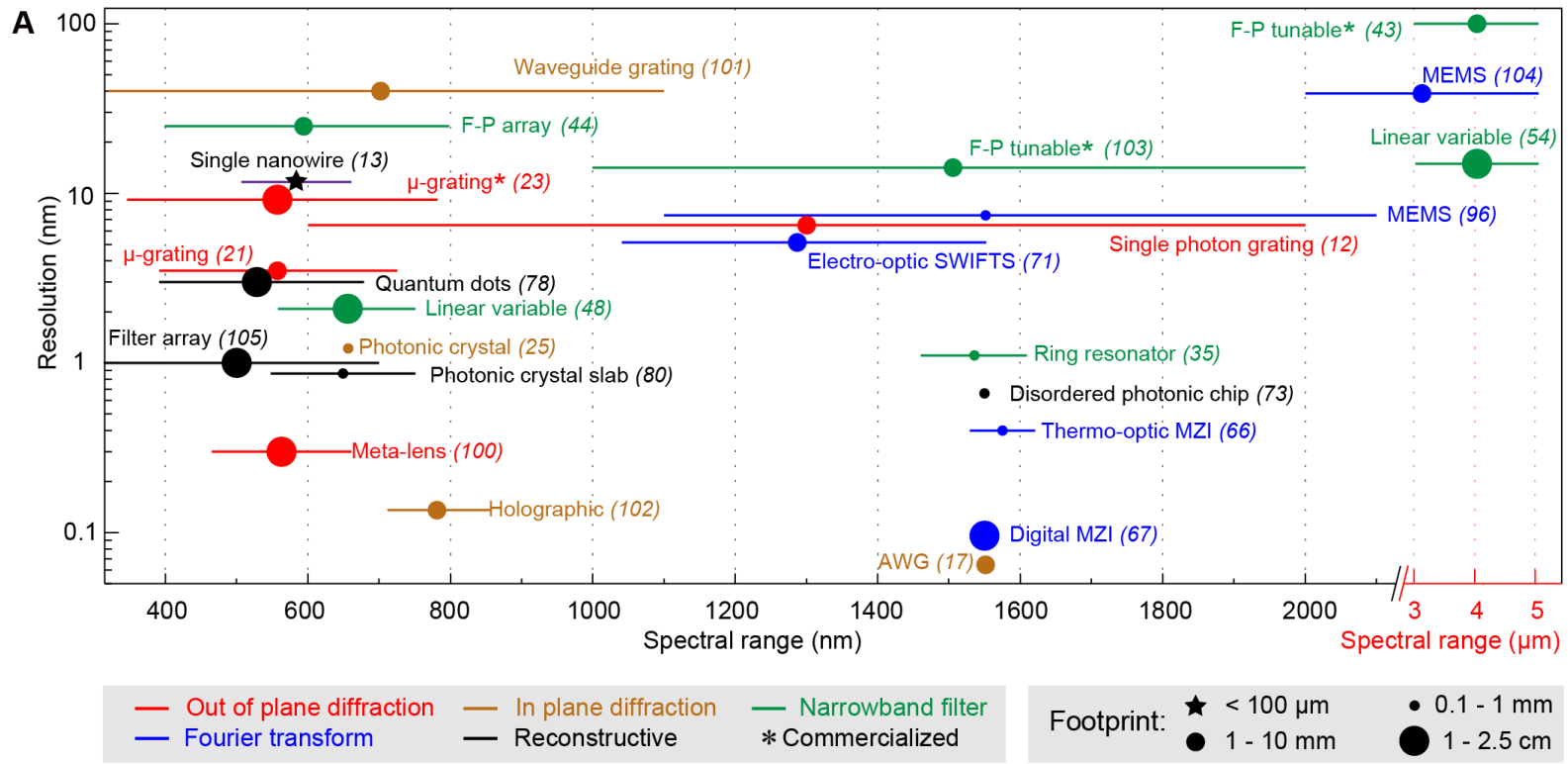

B

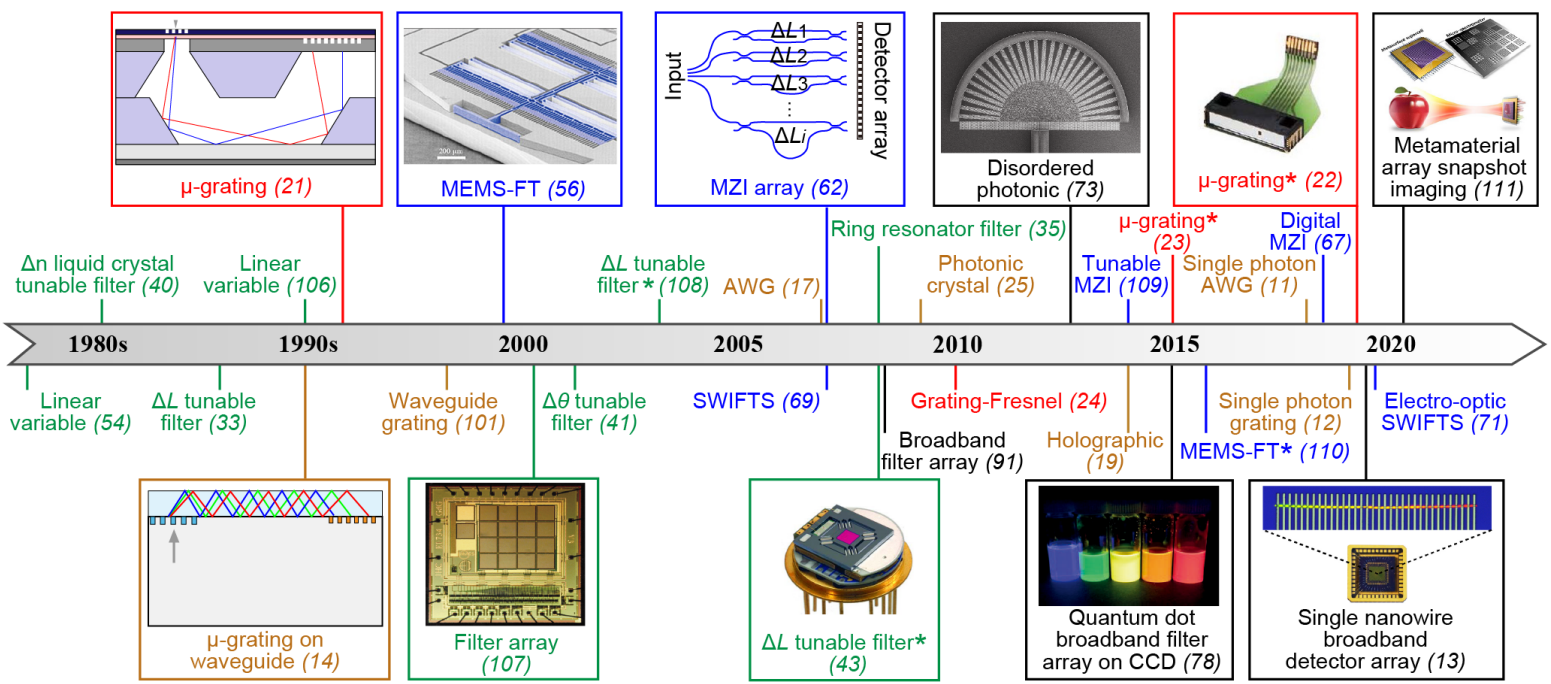

Figure 6. The field of miniaturized spectroscopic devices. (A) Plot comparing the resolution, operational spectral range and footprint for selected device demonstrations in the literature and those that are commercially available (indicated by asterisks), as categorized into their respective sub-fields (see color key). Footprint encompasses those elements of the device that are active in resolving and detecting light, and does not include accessory components such as the read-out electronics or packaging. Reference numbers are indicated within brackets. Note that the spectral range for refs (17), (25), (67) and (73) (which are obscured by the resolution data points) are 10,10,20 and $25 \mathrm{~nm}$ respectively. (B) Timeline illustrating 
the emergence of different technological platforms for microspectrometer systems from the 1980s to present day, sorted by sub-field as displayed in the color key in part A. (Figures have either been redrawn, or adapted with permission, from the references indicated within brackets.)

However, in the last decade, sharp increases in computational power, reductions in processor price and size, have seen attention shift toward spectral reconstruction schemes based on relatively simple, and often disordered, device frameworks. We believe these systems represent the most promising paradigm as their performance can be improved not only by augmenting their hardware but, sometimes more straightforwardly, by optimizing the software that powers them. It

10 seems likely that this trend will continue through further development and optimization of machine learning-based techniques, where the computational power of the accompanying processing systems can shoulder much of the burden for enhancing spectral resolution. As these spectral reconstruction algorithms mature, they will increasingly be able to compensate for the compromises in detector performance necessitated with further miniaturization, allowing for ultra15 compact, yet high performance systems. Clear hurdles still remain in this respect. For example, deep learning algorithms typically require very large, labelled datasets for proper training of the neural networks used, in order to establish an accurate relationship between the measurements and the spectrum to be reconstructed. In cases where it is challenging to produce sufficient, high quality training data, recent developments in transfer learning $(112,113)$ may provide a solution. Here,

20 knowledge learned from a different but related task is utilized to improve the performance of the deep learning algorithm for a target task where sufficient training data is not available.

As well as producing more powerful software, further optimization of device hardware must progress in tandem with design of these algorithms, taking into consideration the factors that are most significantly detrimental to their performance. In reconstructive spectrometers, a key issue is noise - that is, inconsistencies between like measurements of the same spectrum. As discussed previously, thermal and environmental sensitivity must be carefully controlled. As made evident by the equation sets in the previous section, more accurate reconstructions should be possible by increasing the number of detectors and the diversity of the spectral response functions, both of which present their own engineering challenges for consideration in the device 30 architecture. Furthermore, for commercial viability, miniaturization of the CCD or CMOS sensors that accompany the filter arrays, as well as associated read-out and processing electronics, must also be factored in.

\section{Application outlook}

As also seen in Fig. 6, some platforms have already reached full commercial development, showcasing market demand for microspectrometer systems. Over the last two decades, miniaturized, MEMS-based tunable filters and Fourier transform spectrometers as well as a centimeter-scale, packaged, grating-based systems have all become commercially available (3, 22 , 23, 43, 108, 110). These systems can all be produced with well-established semiconductor device fabrication infrastructure; emerging systems that also fit into these frameworks (such as those based on photonic crystals) have a clear advantage over those where novel manufacturing processes must be developed, such as for nanomaterial-based devices.

More recently, the development of spectral sensing systems embedded within smartphones has been evidenced, at CES (consumer electronics show) 2017 and in patent applications by major 
smartphone manufacturers, suggesting that an emergence into the public domain may be imminent $(114,115)$. Indeed, the prospect of a device with a footprint suitable for the smartphone paradigm is arguably the most significant factor driving extreme miniaturization of microspectrometer systems; a breakthrough demonstration here could prove pivotal in terms of attracting further attention and investment in this field. While, as yet, no reconstructive systems have reached commercial maturity, the readily available processing power, coupled with the need for systems with minimized footprint and weight, make the smartphone platform an obvious area for these microspectrometers to emerge.

Beyond smartphones, we believe that high-performance spectrometry contained within ultra-miniaturized packaged systems will find applications in a vast range of fields, industries and commercial technologies, from satellites and drones, to wearables and implantable devices, from chemical and food manufacturing through to cellular imaging and lab-on-a-chip systems. The size of ultra-miniaturized devices $(<100 \mu \mathrm{m})$ alone may make them suitable for wearable or flexible technology, given the comparatively large bending radii expected in operation. Array-based devices which already feature flexible, thin-film filters (78) could prove promising in this regard if the rigid detectors beneath could be replaced with emerging, flexible counterparts (116). Furthermore, bandgap engineering of solution processable materials $(89,90)$ could offer a route toward printable, conformable spectrometers.

Perhaps most exciting are the possibilities for hyperspectral imaging applications involving the simultaneous capture of spectral and spatial information in a 'data cube' with dimensions $(x$, $y, \lambda$ ). Here a long-term goal is a miniaturized, portable 'snapshot' spectral imager with high spectral and spatial resolution; that is, a camera whereby each pixel holds its own, high performance spectrometer. Such a system would be a dramatic advance on many current strategies in development which involve scanning systems with movable parts, or digital mirror device-based spatial light modulators (117). Given their simple (and usually planar) device frameworks, as the active sensing area of computational microspectrometers shrinks toward the $\sim 10 \mu \mathrm{m}$ scale, miniaturized snapshot imagers based on such concepts become increasingly feasible. Indeed, successful prototypes based on arrays of 'super pixels', each containing their own filter array, have very recently been reported $(111,118)$. The addition of a diffuser here can convey a multiplexing advantage whereby each point in the object plane is mapped to many different points (rather than one point, as with a lens) at the super pixel array (118). Besides consumer technology, such devices could potentially revolutionize existing hyperspectral imaging applications in agriculture and mineralogy.

\section{References and Notes:}

1. C. P. Bacon, Y. Mattley, R. DeFrece, Miniature spectroscopic instrumentation: applications to biology and chemistry. Rev. Sci. Instrum. 75, 1 (2004).

2. L. P. Schuler, J. S. Milne, J. M. Dell, L. Faraone, MEMS-based microspectrometer technologies for NIR and MIR wavelengths. J. Phys. D: Appl. Phys 42, 133001 (2009). 
3. J. Malinen et al., Advances in miniature spectrometer and sensor development. Proc. SPIE 9101, 91010C (2014).

4. M. Ebermann et al., Tunable MEMS Fabry-Pérot filters for infrared microspectrometers: a review. Proc. SPIE 9760, 97600H (2016).

5. R. Crocombe, Portable spectroscopy. Appl. Spectrosc. 72, 1701-1751 (2018).

6. R. F. Wolffenbuttel, MEMS-based optical mini- and microspectrometers for the visible and infrared spectral range. J. Micromech. Microeng. 15, S145-S152 (2005).

7. M. Manley, Near-infrared spectroscopy and hyperspectral imaging: non-destructive analysis of biological materials. Chem. Soc. Rev. 43, 8200-8214 (2014).

8. A. J. S. McGonigle et al., Smartphone spectrometers. Sensors 18, (2018).

9. P. Edwards et al., Smartphone based optical spectrometer for diffusive reflectance spectroscopic measurement of hemoglobin. Sci. Rep. 7, 12224 (2017).

10. E. Ryckeboer, R. Bockstaele, M. Vanslembrouck, R. Baets, Glucose sensing by waveguide-based absorption spectroscopy on a silicon chip. Biomed. Opt. Express $\mathbf{5}$, 1636-1648 (2014).

11. V. Kovalyuk et al., On-chip single-photon spectrometer for visible and infrared wavelength range. J. Phys. Conf. Ser. 1124, 051045 (2018).

12. R. Cheng et al., Broadband on-chip single-photon spectrometer. Nat. Commun. 10, 4104 (2019).

13. Z. Yang et al., Single-nanowire spectrometers. Science 365, 1017-1020 (2019).

14. D. S. Goldman, P. L. White, N. C. Anheier, Miniaturized spectrometer employing planar waveguides and grating couplers for chemical analysis. Appl. Opt. 29, 4583-4589 (1990).

15. A. Z. Subramanian et al., Silicon and silicon nitride photonic circuits for spectroscopic sensing on-a-chip. Photonics Res. 3, B47 (2015).

16. D. Sander, J. Muller, Selffocussing phase transmission grating for an integrated optical microspectrometer. Sensor Actuat A: Phys 88, 1-9 (2001).

17. P. Cheben et al., A high-resolution silicon-on-insulator arrayed waveguide grating microspectrometer with sub-micrometer aperture waveguides. Opt. Express 15, 22992306 (2007).

18. B. Gao, Z. Shi, R. W. Boyd, Design of flat-band superprism structures for on-chip spectroscopy. Opt. Express 23, 6491-6496 (2015).

19. G. Calafiore et al., Holographic planar lightwave circuit for on-chip spectroscopy. Light Sci. Appl. 3, e203 (2014).

20. R. F. Wolffenbuttel, State-of-the-art in integrated optical microspectrometers. IEEE Trans. Instrum. Meas. 53, 197-202 (2004).

21. T. A. Kwa, R. F. Wolffenbuttel, Integrated grating/detector array fabricated in silicon using micromachining techniques. Sensor Actuat A: Phys 31, 259-266 (1992).

22. T. Yokino et al., Grating-based ultra-compact SWNIR spectral sensor head developed through MOEMS technology. Proc. SPIE 10931, 8 (2019).

23. Hamamatsu mini-spectrometer micro series C12666MA. https://www.hamamatsu.com/jp/en/product/type/C12666MA/index.html, (2015).

24. C. Yang, K. Shi, P. Edwards, Z. Liu, Demonstration of a PDMS based hybrid grating and Fresnel lens (G-Fresnel) device. Opt. Express 18, 23529-23534 (2010).

25. B. Momeni, E. S. Hosseini, A. Adibi, Planar photonic crystal microspectrometers in silicon-nitride for the visible range. Opt. Express 17, 17060-17069 (2009). 
26. J. J. He et al., Monolithic integrated wavelength demultiplexer based on a waveguide Rowland circle grating in InGaAsP/InP. J. Lightwave. Technol. 16, 631-638 (1998).

27. S. Nezhadbadeh, A. Neumann, P. Zarkesh-Ha, S. R. J. Brueck, Chirped-grating spectrometer-on-a-chip. Opt. Express 28, 24501-24510 (2020).

28. M. Faraji-Dana et al., Compact folded metasurface spectrometer. Nat. Commun. 9, 4196 (2018).

29. S. Janz et al., Planar waveguide echelle gratings in silica-on-silicon. IEEE Photon. Technol. Lett. 16, 503-505 (2004).

30. J. Zou, T. Lang, Z. Le, J. J. He, Ultracompact silicon-on-insulator-based reflective arrayed waveguide gratings for spectroscopic applications. Appl. Opt. 55, 3531-3536 (2016).

31. H. Y. Zhang, X. L. Wang, J. Soos, J. Crisp, Design of a miniature solid-state NIR spectrometer. Proc. SPIE 2475, 376-383 (1995).

32. N. Gat, Imaging spectroscopy using tunable filters: a review. Proc. SPIE 4056, 50-64 (2000).

33. S. R. Mallinson, J. H. Jerman, Miniature micromachined Fabry-Perot interferometers in silicon. Electron. Lett. 23, 1041 (1987).

34. J. P. Carmo et al., A review of visible-range Fabry-Perot microspectrometers in silicon for the industry. Opt. Laser. Technol. 44, 2312-2320 (2012).

35. A. Nitkowski, L. Chen, M. Lipson, Cavity-enhanced on-chip absorption spectroscopy using microring resonators. Opt. Express 16, 11930-11936 (2008).

36. P. M. Zavracky, E. Hennenberg, Miniature Fabry Perot spectrometers using micromachining technology. Proceedings of WESCON'95, 325-332 (1995).

37. J. H. Jerman, D. J. Clift, S. R. Mallinson, A miniature Fabry-Perot-interferometer with a corrugated silicon diaphragm support. Sensor Actuat A: Phys 29, 151-158 (1991).

38. W. J. Gunning, P. Yeh, Multiple-cavity infrared electro-optic tunable filter. Proc. SPIE 0202, 21-25 (1980).

39. Y. Yao et al., Design of programmable multi-wavelength tunable filter on lithium niobate. Results Phys. 15, 102741 (2019).

40. W. Gunning, J. Pasko, J. Tracy, A liquid crystal tunable spectral filter: visible and infrared operation. Proc. SPIE 0268, 190-194 (1981).

41. G. Lammel, S. Schweizer, P. Renaud, Microspectrometer based on a tunable optical filter of porous silicon. Sensor Actuat A: Phys 92, 52-59 (2001).

42. J.-H. Liao, W. Wang, C.-J. Chen, C.-J. Yu, M.-C. Wu, Design and fabrication of largearea tunable MOEMS-based shortwave infrared Fabry-Pérot filters. J. Vac. Sci. Technol. $B$ 37, 032002 (2019).

43. N. Neumann, M. Ebermann, S. Kurth, K. Hiller, Tunable infrared detector with integrated micromachined Fabry-Perot filter. J. Micro-Nanolith. MEM. 7, 021004 (2008).

44. J. H. Correia, G. de Graaf, S. H. Kong, M. Bartek, R. F. Wolffenbuttel, Single-chip CMOS optical microspectrometer. Sensor Actuat A: Phys 82, 191-197 (2000).

45. S. W. Wang et al., Concept of a high-resolution miniature spectrometer using an integrated filter array. Opt. Lett. 32, 632-634 (2007).

46. N. K. Pervez et al., Photonic crystal spectrometer. Opt. Express 18, 8277-8285 (2010).

47. A. Tittl et al., Imaging-based molecular barcoding with pixelated dielectric metasurfaces. Science 360, 1105-1109 (2018). 
48. A. Emadi, H. Wu, G. de Graaf, R. Wolffenbuttel, Design and implementation of a sub$\mathrm{nm}$ resolution microspectrometer based on a linear-variable optical filter. Opt. Express 20, 489-507 (2012).

49. R. G. DeCorby, N. Ponnampalam, E. Epp, T. Allen, J. N. McMullin, Chip-scale spectrometry based on tapered hollow Bragg waveguides. Opt. Express 17, 16632-16645 (2009).

50. P. J. Lapray, X. Wang, J. B. Thomas, P. Gouton, Multispectral filter arrays: recent advances and practical implementation. Sensors 14, 21626-21659 (2014).

51. Q. Hang, B. Ung, I. Syed, N. Guo, M. Skorobogatiy, Photonic bandgap fiber bundle spectrometer. Appl. Opt. 49, 4791-4800 (2010).

52. A. Thelen, Circularly wedged optical coatings I: theory. Appl. Opt. 4, 977 (1965).

53. M. Grundmann, Modeling of a waveguide - based UV-VIS-IR spectrometer based on a lateral (In,Ga)N alloy gradient. Phys. Status Solidi A 216, 1900170 (2019).

54. W. A. Hovis, Jr., W. A. Kley, M. G. Strange, Filter wedge spectrometer for field use. Appl. Opt. 6, 1057-1058 (1967).

55. O. Pust, Innovative filter solutions for hyperspectral imaging. Optik \& Photonik 11, 2427 (2016).

56. O. Manzardo, H. P. Herzig, C. R. Marxer, N. F. de Rooij, Miniaturized time-scanning Fourier transform spectrometer based on silicon technology. Opt. Lett. 24, 1705-1707 (1999).

57. M. Erfan et al., On-chip micro-electro-mechanical system Fourier transform infrared (MEMS FT-IR) spectrometer-based gas sensing. Appl. Spectrosc. 70, 897-904 (2016).

58. U. Wallrabe, C. Solf, J. Mohr, J. G. Korvink, Miniaturized Fourier Transform spectrometer for the near infrared wavelength regime incorporating an electromagnetic linear actuator. Sensor Actuat A: Phys 123-124, 459-467 (2005).

59. W. Wang, S. R. Samuelson, J. Chen, H. Xie, Miniaturizing Fourier transform spectrometer with an electrothermal micromirror. IEEE Photon. Technol. Lett. 27, 14181421 (2015).

60. K. Wang, J. Li, D. F. Lu, Z. M. Qi, Algorithmic enhancement of spectral resolution of a $\mathrm{LiNbO}_{3}$ waveguide-based miniature Fourier transform spectrometer. Appl. Spectrosc. 70, 1685-1691 (2016).

61. P. Malara et al., A self-operating broadband spectrometer on a droplet. Nat. Commun. 11, 2263 (2020).

62. M. Florjanczyk et al., Multiaperture planar waveguide spectrometer formed by arrayed Mach-Zehnder interferometers. Opt. Express 15, 18176-18189 (2007).

63. A. V. Velasco et al., High-resolution Fourier-transform spectrometer chip with microphotonic silicon spiral waveguides. Opt. Lett. 38, 706-708 (2013).

64. H. Podmore et al., Demonstration of a compressive-sensing Fourier-transform on-chip spectrometer. Opt. Lett. 42, 1440-1443 (2017).

65. M. Souza, A. Grieco, N. C. Frateschi, Y. Fainman, Fourier transform spectrometer on silicon with thermo-optic non-linearity and dispersion correction. Nat. Commun. 9, 665 (2018).

66. S. N. Zheng et al., Microring resonator-assisted Fourier transform spectrometer with enhanced resolution and large bandwidth in single chip solution. Nat. Commun. 10, 2349 (2019). 
67. D. M. Kita et al., High-performance and scalable on-chip digital Fourier transform spectroscopy. Nat. Commun. 9, 4405 (2018).

68. A. Herrero-Bermello et al., On-chip Fourier-transform spectrometers and machine learning: a new route to smart photonic sensors. Opt. Lett. 44, 5840-5843 (2019).

69. E. le Coarer et al., Wavelength-scale stationary-wave integrated Fourier-transform spectrometry. Nat. Photon. 1, 473-478 (2007).

70. X. Nie, E. Ryckeboer, G. Roelkens, R. Baets, CMOS-compatible broadband copropagative stationary Fourier transform spectrometer integrated on a silicon nitride photonics platform. Opt. Express 25, A409-A418 (2017).

71. D. Pohl et al., An integrated broadband spectrometer on thin-film lithium niobate. Nat. Photon. 14, 24-29 (2020).

72. B. Redding, S. M. Popoff, H. Cao, All-fiber spectrometer based on speckle pattern reconstruction. Opt. Express 21, 6584-6600 (2013).

73. B. Redding, S. F. Liew, R. Sarma, H. Cao, Compact spectrometer based on a disordered photonic chip. Nat. Photon. 7, 746-751 (2013).

74. W. Hartmann et al., Waveguide - Integrated Broadband Spectrometer Based on Tailored Disorder. Adv. Opt. Mater. 8, 1901602 (2020).

75. B. Redding, S. Fatt Liew, Y. Bromberg, R. Sarma, H. Cao, Evanescently coupled multimode spiral spectrometer. Optica 3, 956 (2016).

76. T. Yang et al., Miniature spectrometer based on diffraction in a dispersive hole array. Opt. Lett. 40, 3217-3220 (2015).

77. P. Wang, R. Menon, Computational spectrometer based on a broadband diffractive optic. Opt. Express 22, 14575-14587 (2014).

78. J. Bao, M. G. Bawendi, A colloidal quantum dot spectrometer. Nature 523, 67-70 (2015).

79. X. Zhu et al., Broadband perovskite quantum dot spectrometer beyond human visual resolution. Light Sci. Appl. 9, (2020).

80. Z. Wang et al., Single-shot on-chip spectral sensors based on photonic crystal slabs. Nat. Commun. 10, 1020 (2019).

81. Y. August, A. Stern, Compressive sensing spectrometry based on liquid crystal devices. Opt. Lett. 38, 4996-4999 (2013).

82. E. Huang, Q. Ma, Z. Liu, Etalon array reconstructive spectrometry. Sci. Rep. 7, 40693 (2017).

83. A. Ahamad et al., Smart nanophotonics silicon spectrometer array for hyperspectral imaging. CLEO, STh3M.2 (2020).

84. Y. Gao et al., Photon-trapping microstructures enable high-speed high-efficiency silicon photodiodes. Nat. Photon. 11, 301-308 (2017).

85. B. Craig, V. R. Shrestha, J. Meng, J. J. Cadusch, K. B. Crozier, Experimental demonstration of infrared spectral reconstruction using plasmonic metasurfaces. Opt. Lett. 43, 4481-4484 (2018).

$40 \quad 86 . \quad$ J. Meng, J. J. Cadusch, K. B. Crozier, Detector-only spectrometer based on structurally colored silicon nanowires and a reconstruction algorithm. Nano Lett. 20, 320-328 (2020).

87. B. Zheng et al., On-chip measurement of photoluminescence with high sensitivity monolithic spectrometer. Adv. Opt. Mater., 2000191 (2020).

88. J. Dong, The smallest nanowire spectrometers. Front. Optoelectron. 12, 341-341 (2020). 
89. M.-N. Zhang et al., Spectrum projection with a bandgap-gradient perovskite cell for colour perception. Light Sci. Appl. 9, 162 (2020).

90. H. Sun et al., In Situ Formed Gradient Bandgap-Tunable Perovskite for Ultrahigh-Speed Color/Spectrum-Sensitive Photodetectors via Electron-Donor Control. Adv. Mater., e1908108 (2020).

91. C. C. Chang, H. N. Lee, On the estimation of target spectrum for filter-array based spectrometers. Opt. Express 16, 1056-1061 (2008).

92. P. C. Hansen, Rank-Deficient and Discrete Ill-Posed Problems. Rank-Deficient and Discrete Ill-Posed Problems (Siam, 2005), vol. 4.

93. C.-C. Chang, H.-Y. Lin, Spectrum reconstruction for on-chip spectrum sensor array using a novel blind nonuniformity correction method. IEEE Sens. J. 12, 2586-2592 (2012).

94. Z. Wang, Z. Yu, Spectral analysis based on compressive sensing in nanophotonic structures. Opt. Express 22, 25608-25614 (2014).

95. S. Zhang, Y. Dong, H. Fu, S. L. Huang, L. Zhang, A spectral reconstruction algorithm of miniature spectrometer based on sparse optimization and dictionary learning. Sensors $\mathbf{1 8}$, 644 (2018).

96. Y. M. Eltagoury, Y. M. Sabry, D. A. Khalil, All-silicon double-cavity Fourier-transform infrared spectrometer on-chip. Adv. Mater. Technol. 4, 1900441 (2019).

97. R. Mannila et al., Gas detection with microelectromechanical Fabry-Perot interferometer technology in cell phone. Proc. SPIE 9482, 94820P (2015).

98. J. H. Correia, M. Bartek, R. F. Wolffenbuttel, High-selectivity single-chip spectrometer in silicon for operation at visible part of the spectrum. IEEE T. Electron Devices 47, 553 559 (2000).

99. A. Herrero-Bermello et al., Temperature dependence mitigation in stationary Fouriertransform on-chip spectrometers. Opt. Lett. 42, 2239-2242 (2017).

100. A. Y. Zhu et al., Ultra-compact visible chiral spectrometer with meta-lenses. $A P L$ Photonics 2, 036103 (2017).

101. D. Sander, M. O. Ducker, O. Blume, J. Muller, An optical microspectrometer in SiONslab-waveguides. Proc. SPIE 2686, 100-107 (1996).

102. A. Koshelev et al., Combination of a spectrometer-on-chip and an array of Young's interferometers for laser spectrum monitoring. Opt. Lett. 39, 5645-5648 (2014).

103. J. Antila et al., MEMS and piezo actuator-based Fabry-Perot interferometer technologies and applications at VTT. Proc. SPIE 7680, 76800U (2010).

104. T. Sandner, A. Kenda, C. Drabe, H. Schenk, W. Scherf, Miniaturized FTIR-spectrometer based on optical MEMS translatory actuator. Proc. SPIE 6466, 646602 (2007).

105. J. Oliver, W. B. Lee, H. N. Lee, Filters with random transmittance for improving resolution in filter-array-based spectrometers. Opt. Express 21, 3969-3989 (2013).

106. A. M. Mika, Linear-wedge spectrometer. Proc. SPIE 1298, 127-131 (1990).

107. S. H. Kong, J. H. Correia, G. de Graaf, M. Bartek, R. F. Wolffenbuttel, Integrated silicon microspectrometers. IEEE Instru. Meas. Mag. 4, 34-38 (2001).

108. P. Kotidis et al., Optical, tunable filter-based micro-instrumentation for industrial applications. Proc. Instr. Syst. and Autom. Soc.(ISA), 1-14 (2003).

109. J. Li, D. F. Lu, Z. M. Qi, Miniature Fourier transform spectrometer based on wavelength dependence of half-wave voltage of a $\mathrm{LiNbO}_{3}$ waveguide interferometer. Opt. Lett. 39, 3923-3926 (2014). 
110. NeoSpectra-micro FTIR. https://www.neospectra.com/our-offerings/neospectra-micro/, (2016).

111. X. Cai et al., One-shot ultraspectral imaging with reconfigurable metasurfaces. http://arxiv.org/abs/2005.02689, (2020).

112. D. Jha et al., Enhancing materials property prediction by leveraging computational and experimental data using deep transfer learning. Nat. Commun. 10, 5316 (2019).

113. J. S. Smith et al., Approaching coupled cluster accuracy with a general-purpose neural network potential through transfer learning. Nat. Commun. 10, 2903 (2019).

114. Changhong $\mathrm{H} 2$ spectrometer phone. https://www.cnet.com/pictures/changhong-h2-ces2017/3/, (2017).

115. Electronic device comprising plurality of light sources. US Patent 20190290148A1, (2018).

116. A. Koppelhuber, O. Bimber, Towards a transparent, flexible, scalable and disposable image sensor using thin-film luminescent concentrators. Opt. Express 21, 4796-4810 (2013)

117. N. Hagen, M. W. Kudenov, Review of snapshot spectral imaging technologies. Opt. Eng. 52, 090901 (2013).

118. K. Monakhova, K. Yanny, N. Aggarwal, L. Waller, Spectral DiffuserCam: lensless snapshot hyperspectral imaging with a spectral filter array. Optica 7, 1298 (2020).

\section{Acknowledgments}

We thank W. Han for valuable discussions. Funding: The authors would like to acknowledge funding support from National Natural Science Foundation of China (51706141, 51976122) and EPSRC (EP/L016087/1). Author contributions: Z.Y. and T.A.O. contributed equally to this work. Competing interests: Authors declare no competing interests. 